PROCEEDINGS OF THE

AMERICAN MATHEMATICAL SOCIETY

Volume 134, Number 8, Pages 2349-2358

S 0002-9939(06)08268-2

Article electronically published on February 17, 2006

\title{
IDENTIFICATION OF SOME REAL INTERPOLATION SPACES
}

\author{
MARKUS HAASE
}

(Communicated by N. Tomczak-Jaegermann)

\begin{abstract}
We interrelate the real interpolation spaces associated with the couples $(X, Y),(X+Y, Y),(X, X \cap Y)$, and $(X+Y, X \cap Y)$, proving among others the identities

$$
\begin{aligned}
(X+Y, X)_{\theta, p} \cap(X+Y, Y)_{\theta, p} & =(X+Y, X \cap Y)_{\theta, p}, \\
(X+Y, X)_{\theta, p} \cap(X+Y, Y)_{1-\theta, p} & =(X, Y)_{\theta, p}, \\
(X, X \cap Y)_{\theta, p}+(Y, X \cap Y)_{\theta, p} & =(X+Y, X \cap Y)_{\theta, p}, \\
(X, X \cap Y)_{\theta, p}+(Y, X \cap Y)_{1-\theta, p} & =(X, Y)_{\theta, p}
\end{aligned}
$$

for all $p \in[1, \infty], \theta \in[0,1]$.
\end{abstract}

\section{INTRODUCTION AND MAIN RESUlts}

Since its invention in the late 1950's, the theory of interpolation spaces has seen a tremendous development with various applications in different fields of mathematics. Several monographs ([8, [2], [15, 3]) have appeared during the last five decades comprising what can now be regarded as standard knowledge. As is frequent with monographs, they foster the impression that on the theoretical side at least the elementary questions are solved.

However, it seems that a very natural question of interpolation theory has not been made sufficiently clear so far, namely the problem of how the interpolation spaces for the couples $(X, Y),(X+Y, X),(X, X \cap Y)$ and $(X+Y, X \cap Y)$ interrelate. (Partial results can be found in [10] and [12.) The purpose of this note is to give a somewhat complete answer to this question in the case of real interpolation.

In the remainder of this section we will introduce some notation and state the main results. Section 2 is devoted to proofs. Finally, in Section 3 we sketch some applications to the so-called intersection problem and to the theory of sectorial operators.

In the following, $(X, Y)$ will always denote a Banach couple, i.e., a pair of Banach spaces $X, Y$ which are tacitly assumed to be continuously embedded into a bigger Banach space. For $p \in[1, \infty], \theta \in[0,1]$ we construct the real interpolation spaces

Received by the editors October 20, 2004 and, in revised form, March 8, 2005.

2000 Mathematics Subject Classification. Primary 47A60, 47D06.

Key words and phrases. Interpolation space, K-method, real method of interpolation, intersection property, sectorial operator.

The author gratefully acknowledges the financial support from the EU-Research Training Network "Evolution Equations for Deterministic and Stochastic Systems", Contract No. HPRN-CT2002-00281.

(C)2006 American Mathematical Society Reverts to public domain 28 years from publication 
$(X, Y)_{\theta, p}$ via the so-called "K-method". The "K-functional" is defined by

$$
K(t, x, X, Y):=\inf \left\{\|x\|_{X}+t\|b\|_{Y} \mid x=a+b\right\}
$$

for $x \in X+Y, t>0$. Let us use the symbol $\mathbf{L}_{*}^{p}(a, b)$ to denote the space of (equivalence classes of) functions on the interval $(a, b) \subset(0, \infty)$ which are $p$-integrable with respect to the measure $d t / t$. Here we simply write $t:=(t \mapsto t)$ for the positive real coordinate. With this notation, the real interpolation spaces are defined by

$$
(X, Y)_{\theta, p}=\left\{x \in X+Y \mid t^{-\theta} K(t, x, X, Y) \in \mathbf{L}_{*}^{\boldsymbol{p}}(0, \infty)\right\}
$$

with norm $\|K(\cdot, x, X, Y)\|_{\mathbf{L}_{*}^{p}(0, \infty)}$. We will use the identities

$$
K(t, x, X, Y)=t K\left(t^{-1}, x, Y, X\right) \quad \text { and } \quad(X, Y)_{\theta, p}=(Y, X)_{1-\theta, p}
$$

but no method other than the K-method is used in this paper. One can consult any of the above-mentioned books for more information, but we also recommend the lecture notes [9]. For $\theta \in[0,1]$ we abbreviate $\bar{\theta}=\max (\theta, 1-\theta)$ and $\underline{\theta}=\min (\theta, 1-\theta)$. Since the case $1 \leq p<\infty, \theta \in\{0,1\}$ is in a sense "degenerate", we introduce the set

$$
\Gamma:=([0,1] \times[1, \infty]) \backslash(\{0,1\} \times[1, \infty)) .
$$

We are now ready to state the main result.

Theorem 1.1. Let $(X, Y)$ be a Banach couple and let $(\theta, p) \in \Gamma$. Then the following identities hold:

$$
\begin{aligned}
(X+Y, Y)_{\theta, p} \cap X=(X, Y)_{\theta, p} \cap X & =(X, X \cap Y)_{\theta, p}, \\
(X \cap Y, Y)_{\theta, p}+X=(X, Y)_{\theta, p}+X & =(X, X+Y)_{\theta, p}, \\
(X+Y, Y)_{\theta, p} \cap(X, X+Y)_{\theta, p} & =(X, Y)_{\theta, p}, \\
(X \cap Y, Y)_{\theta, p}+(X, X \cap Y)_{\theta, p} & =(X, Y)_{\theta, p}, \\
(X+Y, X)_{\theta, p} \cap(X+Y, Y)_{\theta, p} & =(X+Y, X \cap Y)_{\theta, p}, \\
(X, X \cap Y)_{\theta, p}+(Y, X \cap Y)_{\theta, p} & =(X+Y, X \cap Y)_{\theta, p}, \\
(X, Y)_{\theta, p} \cap(X, Y)_{1-\theta, p} & =(X+Y, X \cap Y)_{\bar{\theta}, p}, \\
(X, Y)_{\theta, p}+(X, Y)_{1-\theta, p} & =(X+Y, X \cap Y)_{\underline{\theta}, p}, \\
(X, X \cap Y)_{\theta, p} \cap(X+Y, X \cap Y)_{1-\theta, p} & =(X, X \cap Y)_{\bar{\theta}, p}, \\
(X+Y, Y)_{\theta, p}+(X+Y, X \cap Y)_{1-\theta, p} & =(X+Y, Y)_{\underline{\theta}, p}, \\
(X, X \cap Y)_{\underline{\theta}, p}+(X+Y, X \cap Y)_{\bar{\theta}, p} & =(X, Y)_{\underline{\theta}, p}, \\
(X+Y, X)_{\bar{\theta}, p} \cap(X+Y, X \cap Y)_{\underline{\theta}, p} & =(X, Y)_{\underline{\theta}, p} .
\end{aligned}
$$

The proof will be given in Propositions $2.4-2.10$ of Section 2 ,

Corollary 1.2. Let $(X, Y)$ be a Banach couple and let $(\theta, p) \in \Gamma$. Then:

$$
\begin{gathered}
(X+Y, X \cap Y)_{\bar{\theta}, p} \subset(X, Y)_{\theta, p} \subset(X+Y, X \cap Y)_{\underline{\theta}, p}, \\
(X, Y)_{\theta, p} \in K_{\underline{\theta}}(X+Y, X \cap Y) \cap J_{\bar{\theta}}(X+Y, X \cap Y), \\
(X, Y)_{\frac{1}{2}, p}=(X+Y, X \cap Y)_{\frac{1}{2}, p} .
\end{gathered}
$$


Proof. The first assertion is immediate from Theorem 1.1. The other two assertions follow readily from the first.

It is clear that Theorem 1.1 does not at all clarify "what's going on". Therefore we will now give a geometric illustration of the above theorem. Let $A$ be a vector space and let $B, C, D \subset A$ be subspaces of $A$ with $D \subset B \cap C$. Such a situation we will depict in a diagram

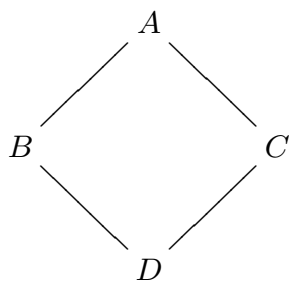

and we call this diagram an (elementary) block. We call the above elementary block proper if $A=B+C$ and $D=B \cap C$. Thus, (1.7) and (1.8) of Theorem 1.1 say that if $\theta \leq \frac{1}{2}$ the block

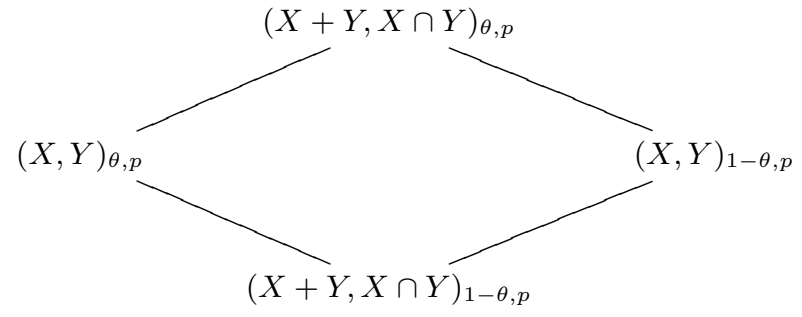

is proper. Now, again supposing $\theta \leq \frac{1}{2}$, consider the following diagram:

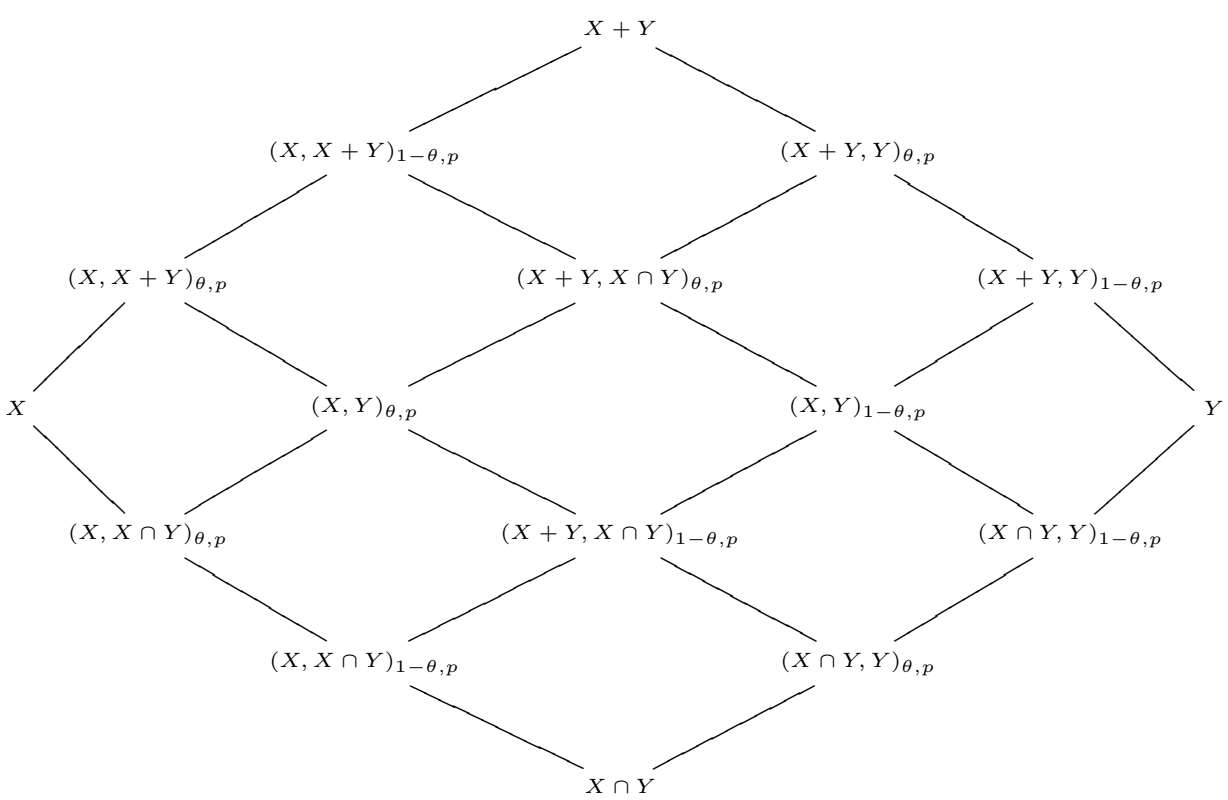

Then we can summarize the results of Theorem 1.1 as follows. 
Theorem 1.3. Let $(X, Y)$ be any Banach couple and let $(\theta, p) \in \Gamma$ with $\theta \leq \frac{1}{2}$. Then in the above diagram all elementary blocks are proper.

Proof. The statement follows directly from Theorem 1.1, where one sometimes has to employ the symmetry property $(A, B)_{\theta, p}=(B, A)_{1-\theta, p}$. More precisely, formulae (1.1) and (1.2) account for the leftmost block. For the block one step in direction north-east one uses (1.11) and (1.12), whereas (1.9) and (1.10) are needed for the block below this. So, the three leftmost blocks are done. The statement for the three rightmost blocks follows by interchanging the roles of $X$ and $Y$. Equations (1.5) and (1.6) do the job for the highest and lowest blocks, and the block in the middle is dealt with by the identities (1.7) and (1.8).

Let us state another consequence.

Theorem 1.4. Let $(X, Y)$ be any Banach couple and let $(\theta, p) \in \Gamma$. Then the identities

$$
\begin{aligned}
\left((X, X \cap Y)_{\theta, p},(X+Y, Y)_{\theta, p}\right)_{\theta, p} & =(X, Y)_{\theta, p}, \\
\left((X, X \cap Y)_{\theta, p},(X+Y, Y)_{\theta, p}\right)_{1-\theta, p} & =(X+Y, X \cap Y)_{\theta, p}
\end{aligned}
$$

hold.

Proof. Since $(X, X \cap Y)_{\theta, p} \subset X$ and $(X+Y, Y)_{\theta, p} \subset X+Y$, we have

$$
\begin{aligned}
& \left((X, X \cap Y)_{\theta, p},(X+Y, Y)_{\theta, p}\right)_{\theta, p} \subset(X, X+Y)_{\theta, p} \cap(X+Y, Y)_{\theta, p} \\
& \quad=(X, Y)_{\theta, p}=(X, X \cap Y)_{\theta, p}+(X \cap Y, Y)_{\theta, p} \\
& \quad \subset\left((X, X \cap Y)_{\theta, p},(X+Y, Y)_{\theta, p}\right)_{\theta, p},
\end{aligned}
$$

where we have used (1.3) and (1.4) for the equalities in the middle. The proof of the second assertion is similar. Note that in the degenerate case $(\theta, p) \notin \Gamma$, the equations hold trivially.

Corollary 1.5. Let $(X, Y)$ be any Banach couple and let $(\theta, p) \in \Gamma$ with $\theta \leq \frac{1}{2}$. Then

$$
\left((X+Y, X \cap Y)_{\theta, p},(X, X \cap Y)_{\theta, p}\right)_{\frac{1-2 \theta}{1-\theta}, p}=(X, Y)_{\theta, p} .
$$

Proof. Apply the Reiteration Theorem (see, e.g., [2, Thm. 3.5.3] or [9, Remark 1.3.6]) and Theorem 1.4.

\section{Proof of Theorem 1.1}

We will start with a couple of lemmas.

Lemma 2.1. Let $(X, Y)$ be a Banach couple and $x \in X+Y$. Then

$$
K(t, x, X, Y)=K(t, x, X, X+Y) \quad(t \geq 1)
$$

Proof. Let $x=a+b$ with $a \in X, b \in X+Y$ and let $b=c+d$ with $c \in X, d \in Y$. Then $K(t, x, X, Y) \leq\|a+c\|_{X}+t\|d\|_{Y} \leq\|a\|_{X}+\|c\|_{X}+t\|d\|_{Y} \leq\|a\|_{X}+t\left[\|c\|_{X}+t\|d\|_{Y}\right]$ for $t \geq 1$. Taking the infimum with respect to the decompositions $b=c+d$ we obtain $K(t, x, X, Y) \leq\|a\|_{X}+t\|b\|_{X+Y}$, and going over to the infimum again yields $K(t, x, X, Y) \leq K(t, x, X, X+Y)$. The reverse inequality $K(t, x, X, X+Y) \leq$ $K(t, x, X, Y)$ holds trivially for all $t>0$. 
Recall that if $(A, B)$ is a Banach couple and $A \subset B$, then for having $a \in(A, B)_{\theta, p}$ only the behaviour of $K(t, a, A, B)$ on $(1, \infty)$ is relevant and

$$
\left\|t^{-\theta} K(t, a, A, B)\right\|_{\mathbf{L}_{*}^{p}(1, \infty)}
$$

is an equivalent norm on $(A, B)_{\theta, p}$. An analogous remark applies in the case $B \subset A$.

Lemma 2.2. Let $(X, Y)$ be a Banach couple and $(\theta, p) \in \Gamma$. Then

$$
\begin{aligned}
(X, X+Y)_{\theta, p} & =\left\{x \in X+Y \mid t^{-\theta} K(t, x, X, Y) \in \mathbf{L}_{*}^{\boldsymbol{p}}(1, \infty)\right\}, \\
(X+Y, Y)_{\theta, p} & =\left\{x \in X+Y \mid t^{-\theta} K(t, x, X, Y) \in \mathbf{L}_{*}^{\boldsymbol{p}}(0,1)\right\} .
\end{aligned}
$$

Proof. This is a straightforward application of Lemma 2.1 and the remarks preceding this lemma.

The next one is the final auxiliary result.

Lemma 2.3. Let $(X, Y)$ be a Banach couple and $x \in X$. Then

$$
\begin{aligned}
K(t, x, X, Y) & \leq K(t, x, X, X \cap Y) \quad(t>0), \\
K(t, x, X, X \cap Y) & \leq 2 K(t, x, X, Y)+t\|x\|_{X} \quad(t \leq 1) .
\end{aligned}
$$

Proof. The first assertion holds trivially. To prove the second, take $x=a+b$, where $a \in X, b \in Y$. Then obviously $b \in X \cap Y$ and $K(t, x, X, X \cap Y) \leq\|a\|_{X}+t\|b\|_{X \cap Y}=$ $\|a\|_{X}+t\|b\|_{X}+t\|b\|_{Y} \leq\|a\|_{X}+t\|a\|_{X}+t\|x\|_{X}+t\|b\|_{Y} \leq 2\|a\|_{X}+t\|b\|_{Y}+t\|x\|_{X}$. Taking the infimum yields $K(t, x, X, X \cap Y) \leq 2 K(t, x, X, Y)+t\|x\|_{X}$.

Proposition 2.4. Let $(X, Y)$ be a Banach couple and $(\theta, p) \in \Gamma$. Then the following identities hold:

$$
\begin{aligned}
(X+Y, Y)_{\theta, p} \cap X & =(X, Y)_{\theta, p} \cap X=(X, X \cap Y)_{\theta, p}, \\
(X \cap Y, Y)_{\theta, p}+X & =(X, Y)_{\theta, p}+X=(X, X+Y)_{\theta, p} .
\end{aligned}
$$

Proof. Let us prove the identity (1.1). The chain of inclusions " $\supset$ " is clear, hence we have to show $(X+Y, Y)_{\theta, p} \cap X \subset(X, X \cap Y)_{\theta, p}$. Take $x \in(X+Y, Y)_{\theta, p} \cap X$. Since $x \in X$, only the behaviour of $K(t, x, X, X \cap Y)$ on $(0,1)$ matters. According to Lemma 2.3 and Lemma 2.1

$$
\begin{aligned}
K(t, x, X, X \cap Y) & \leq 2 K(t, x, X, Y)+t\|x\|_{X}=2 t K\left(t^{-1}, x, Y, X\right)+t\|x\|_{X} \\
& =2 t K\left(t^{-1}, x, Y, X+Y\right)+t\|x\|_{X} \\
& =2 K(t, x, X+Y, Y)+t\|x\|_{X} .
\end{aligned}
$$

Now, the identity (1.1) follows.

To prove the identity (1.2), we note as before that one chain of inclusions is trivial. Take $x \in(X, X+Y)_{\theta, p}$ and write $x=a+b$ with $a \in X, b \in Y$. Then by Lemma 2.1] we have

$$
K(t, b, X, Y) \leq K(t, x, X, Y)+K(t, a, X, Y) \leq K(t, x, X, X+Y)+\|a\|_{X}
$$

for $t \geq 1$ and $K(t, b, X, Y) \leq t\|b\|_{Y}$ for $t \leq 1$. Hence we have $b \in(X, Y)_{\theta, p}$.

Remark 2.5. In our proofs we usually only care for set-theoretic inclusions and not for norm inequalities. These are automatic by the Closed Graph Theorem. However, a careful analysis of our arguments would also lead to norm inequalities. 
E.g., arguing a bit further in the last proof would lead to a constant $C$ such that

$$
\|x\|_{X+(X, Y)_{\theta, p}} \leq C\|x\|_{(X, X+Y)_{\theta, p}}
$$

for all $x \in(X, X+Y)_{\theta, p}$.

In the proof of the next result we will have occasion to use the so-called modular law for vector subspaces $A, B, C$ of a vector space $Z$ :

$$
B \subset C \quad \Longrightarrow \quad(A+B) \cap C=(A \cap C)+B .
$$

The proof of this fact is trivial.

Proposition 2.6. Let $(X, Y)$ be a Banach couple and $(\theta, p) \in \Gamma$. Then the following identities hold:

$$
\begin{aligned}
(X+Y, Y)_{\theta, p} \cap(X, X+Y)_{\theta, p} & =(X, Y)_{\theta, p}, \\
(X \cap Y, Y)_{\theta, p}+(X, X \cap Y)_{\theta, p} & =(X, Y)_{\theta, p} .
\end{aligned}
$$

Proof. The first identity is straightforward from Lemma 2.2 and the definition of $(X, Y)_{\theta, p}$. Let us prove the second. As usual, one inclusion is trivial. To establish the other inclusion, take $x \in(X, Y)_{\theta, p}$ and write $x=a+b$ with $a \in X, b \in Y$. Then

$$
\begin{aligned}
b & =x-a \in\left[X+(X, Y)_{\theta, p}\right] \cap Y=\left[X+(X \cap Y, Y)_{\theta, p}\right] \cap Y \\
& =X \cap Y+(X \cap Y, Y)_{\theta, p}=(X \cap Y, Y)_{\theta, p},
\end{aligned}
$$

where we used Proposition 2.4 and the modular law. Analogously (by interchanging the roles of $X$ and $Y$ in Proposition 2.4) one also shows $a \in(X, X \cap Y)_{\theta, p}$. Hence the second identity is proved.

Our next result looks very similar to the one before.

Proposition 2.7. Let $(X, Y)$ be a Banach couple and $(\theta, p) \in \Gamma$. Then the following identities hold:

$$
\begin{aligned}
& (X+Y, X)_{\theta, p} \cap(X+Y, Y)_{\theta, p}=(X+Y, X \cap Y)_{\theta, p}, \\
& (X, X \cap Y)_{\theta, p}+(Y, X \cap Y)_{\theta, p}=(X+Y, X \cap Y)_{\theta, p} .
\end{aligned}
$$

Proof. We use (1.2) together with the modular law and compute:

$$
\begin{aligned}
(X+Y, X)_{\theta, p} \cap & (X+Y, Y)_{\theta, p}=\left[X+(Y, X \cap Y)_{\theta, p}\right] \cap\left[Y+(X, X \cap Y)_{\theta, p}\right] \\
& =\left\{X \cap\left[Y+(X, X \cap Y)_{\theta, p}\right]\right\}+(Y, X \cap Y)_{\theta, p} \\
& =(X \cap Y)+(X, X \cap Y)_{\theta, p}+(Y, X \cap Y)_{\theta, p} \\
& =(X, X \cap Y)_{\theta, p}+(Y, X \cap Y)_{\theta, p} \\
& \subset(X+Y, X \cap Y)_{\theta, p} \subset(X+Y, X)_{\theta, p} \cap(X+Y, Y)_{\theta, p} .
\end{aligned}
$$

Proposition 2.8. Let $(X, Y)$ be a Banach couple and $(\theta, p) \in \Gamma$. Then the following identities hold:

$$
\begin{aligned}
(X, Y)_{\theta, p} \cap(X, Y)_{1-\theta, p} & =(X+Y, X \cap Y)_{\bar{\theta}, p}, \\
(X, Y)_{\theta, p}+(X, Y)_{1-\theta, p} & =(X+Y, X \cap Y)_{\underline{\theta}, p} .
\end{aligned}
$$


Proof. Without restriction we can assume $0 \leq \theta \leq \frac{1}{2}$. Using the identity (1.5) we write

$$
\begin{aligned}
& (X, Y)_{\theta, p} \cap(X, Y)_{1-\theta, p} \subset(X+Y, X)_{1-\theta, p} \cap(X+Y, Y)_{1-\theta, p} \\
& =(X+Y, X \cap Y)_{1-\theta, p}
\end{aligned}
$$

Also, since $\theta \leq 1-\theta$,

$$
\begin{aligned}
(X+Y, X \cap Y)_{1-\theta, p} & \subset(X+Y, X)_{1-\theta, p} \cap(X+Y, Y)_{1-\theta, p} \\
& \subset(X, X+Y)_{\theta, p} \cap(X+Y, Y)_{\theta, p}=(X, Y)_{\theta, p}
\end{aligned}
$$

by Proposition 2.4. Interchanging the roles of $X$ and $Y$ in this inclusion gives $(X+Y, X \cap Y)_{1-\theta, p} \subset(Y, X)_{\theta, p}=(X, Y)_{1-\theta, p}$. Hence, identity (1.7) is completely proved. Let us turn to (1.8). On the one hand, we have

$$
(X+Y, X \cap Y)_{\theta, p}=(X, X \cap Y)_{\theta, p}+(Y, X \cap Y)_{\theta, p} \subset(X, Y)_{\theta, p}+(Y, X)_{\theta, p} .
$$

On the other hand,

$$
\begin{aligned}
(X, Y)_{\theta, p}= & (X, X \cap Y)_{\theta, p}+(X \cap Y, Y)_{\theta, p} \subset(X+Y, X \cap Y)_{\theta, p} \\
& +(X \cap Y, X+Y)_{\theta, p} \subset(X+Y, X \cap Y)_{\theta, p},
\end{aligned}
$$

since $\theta \leq 1-\theta$. This concludes the proof.

Proposition 2.9. Let $(X, Y)$ be a Banach couple and $(\theta, p) \in \Gamma$. Then the following identities hold:

$$
\begin{aligned}
& (X, X \cap Y)_{\theta, p} \cap(X+Y, X \cap Y)_{1-\theta, p}=(X, X \cap Y)_{\bar{\theta}, p}, \\
& (X+Y, Y)_{1-\theta, p}+(X+Y, X \cap Y)_{\theta, p}=(X+Y, Y)_{\underline{\theta}, p} .
\end{aligned}
$$

Proof. Let us prove (1.9). Using (1.6) and the modular law we write

$$
\begin{aligned}
(X, & X \cap Y)_{\theta, p} \cap(X+Y, X \cap Y)_{1-\theta, p} \\
& =(X, X \cap Y)_{\theta, p} \cap\left[(X, X \cap Y)_{1-\theta, p}+(Y, X \cap Y)_{1-\theta, p}\right] \\
& =(X, X \cap Y)_{\theta, p} \cap\left[(X, X \cap Y)_{1-\theta, p}+\left(X \cap(Y, X \cap Y)_{1-\theta, p}\right)\right] \\
& =(X, X \cap Y)_{\theta, p} \cap\left[(X, X \cap Y)_{1-\theta, p}+(X \cap Y)\right] \\
& =(X, X \cap Y)_{\theta, p} \cap(X, X \cap Y)_{1-\theta, p}=(X, X \cap Y)_{\bar{\theta}, p} .
\end{aligned}
$$

To prove (1.10) we use (1.5) and again the modular law (but in reverse direction):

$$
\begin{aligned}
(X & +Y, Y)_{1-\theta, p}+(X+Y, X \cap Y)_{\theta, p} \\
& =(X+Y, Y)_{1-\theta, p}+\left[(X+Y, X)_{\theta, p} \cap(X+Y, Y)_{\theta, p}\right] \\
& =(X+Y, Y)_{1-\theta, p}+\left[\left(Y+(X+Y, X)_{\theta, p}\right) \cap(X+Y, Y)_{\theta, p}\right] \\
& =(X+Y, Y)_{1-\theta, p}+\left[(X+Y) \cap(X+Y, Y)_{\theta, p}\right] \\
& =(X+Y, Y)_{1-\theta, p}+(X+Y, Y)_{\theta, p}=(X+Y, Y)_{\underline{\theta}, p} .
\end{aligned}
$$

Proposition 2.10. Let $(X, Y)$ be a Banach couple and $(\theta, p) \in \Gamma$. Then the following identities hold:

$$
\begin{aligned}
(X, X \cap Y)_{\underline{\theta}, p}+(X+Y, X \cap Y)_{\bar{\theta}, p} & =(X, Y)_{\underline{\theta}, p}, \\
(X+Y, X)_{\bar{\theta}, p} \cap(X+Y, X \cap Y)_{\underline{\theta}, p} & =(X, Y)_{\underline{\theta}, p} .
\end{aligned}
$$


Proof. We can assume without restriction that $\theta \leq \frac{1}{2}$. Then

$$
\begin{aligned}
(X, & X \cap Y)_{\theta, p}+(X+Y, X \cap Y)_{1-\theta, p} \\
& =(X, X \cap Y)_{\theta, p}+(X, X \cap Y)_{1-\theta, p}+(Y, X \cap Y)_{1-\theta, p} \\
& =(X, X \cap Y)_{\theta, p}+(Y, X \cap Y)_{1-\theta, p}=(X, Y)_{\theta, p}
\end{aligned}
$$

by (1.6) and (1.4). Similarly, using (1.5) and (1.3) we obtain

$$
\begin{aligned}
(X & +Y, X)_{1-\theta, p} \cap(X+Y, X \cap Y)_{\theta, p} \\
& =(X+Y, X)_{1-\theta, p} \cap(X+Y, X)_{\theta, p} \cap(X+Y, Y)_{\theta, p} \\
& =(X+Y, X)_{1-\theta, p} \cap(X+Y, Y)_{\theta, p}=(X, Y)_{\theta, p} .
\end{aligned}
$$

\section{Applications}

3.1. Instantiations. Of course, Theorems 1.1 and 1.3 have an immediate meaning for some known interpolation couples. For example, if applied to the couple $\left(\mathbf{L}^{\mathbf{1}}, \mathbf{L}^{\infty}\right)$ one obtains the identity

$$
\mathbf{L}^{1} \cap \mathbf{L}^{\frac{1}{1-\theta}, p}=\left(\mathbf{L}^{1}, \mathbf{L}^{1} \cap \mathbf{L}^{\infty}\right)_{\theta, p},
$$

where $\mathbf{L}^{\frac{1}{1-\theta}, p}$ is the usual Lorentz space. A second instance arises when taking $X=$ $\mathbf{C}^{\mathbf{b}}\left(\mathbb{R}^{d}\right)$ as the Banach space of the continuous and uniformly bounded functions on $\mathbb{R}^{d}$ and $Y=\operatorname{Lip}\left(\mathbb{R}^{d}\right)$ as the space of Lipschitz-continuous functions on $\mathbb{R}^{d}$ with the norm $\|f\|_{\text {Lip }}=[f]_{1}+|f(0)|$. Then $(X, Y)_{\theta, \infty}=\mathbf{C}^{\theta}\left(\mathbb{R}^{d}\right)$ is the space of Hölder-continuous functions of degree $\theta$. Equation (1.1) then yields

$$
\mathbf{C}^{\theta}\left(\mathbb{R}^{d}\right) \cap \mathbf{C}^{\mathbf{b}}\left(\mathbb{R}^{d}\right)=\left(\mathbf{C}^{\mathbf{b}}\left(\mathbb{R}^{d}\right), \mathbf{C}^{\mathbf{b}}\left(\mathbb{R}^{d}\right) \cap \mathbf{L i p}\left(\mathbb{R}^{d}\right)\right)_{\theta, \infty} .
$$

3.2. Sectorial operators. Theorem 1.1 was originally motivated by the study of functional calculus for injective, but not invertible sectorial operators; see [13] and [7] for definition and basic results. There, one has a picture

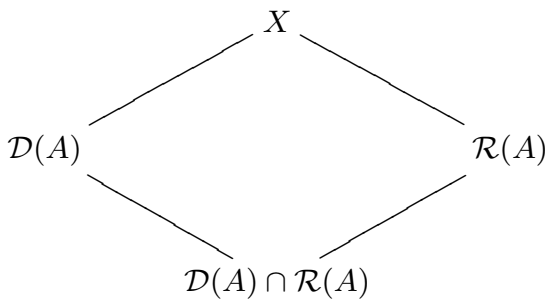

where $X$ is the underlying Banach space, and $\mathcal{D}(A)$ and $\mathcal{R}(A)$ are the domain and the range of the sectorial operator $A$. Now, Dore has shown in 4] that in fact $A$ has a bounded $H^{\infty}$-calculus when restricted to the vertical interpolation spaces $(X, \mathcal{D}(A) \cap \mathcal{R}(A))_{\theta, p}$. However, generalizing ideas from [1] to arbitrary Banach spaces, one can show that $A$ has in fact a bounded $H^{\infty}$-calculus in each of the horizontal spaces $(\mathcal{D}(A), \mathcal{R}(A))_{\theta, p}$. Our Theorem 1.3 now shows that Dore's result is actually a corollary of this.

A second application in connection with sectorial operators appears in characterizing growth conditions like

$$
\sup _{t>0}\left\|t^{\theta} C(t+A)^{-1}\right\|_{Y \rightarrow X}<\infty \quad \text { or } \quad \sup _{t>0}\left\|t^{\theta}(t+A)^{-1} B\right\|_{Y \rightarrow \mathcal{D}(A)}<\infty
$$


by means of interpolation spaces (see [6]). Here, $Y$ is another Banach space and $C: \mathcal{D}(A) \longrightarrow Y$ and $B: Y \longrightarrow X$ are linear operators. The above growth conditions are common assumptions in perturbation results.

3.3. The intersection problem. As another consequence of our results, let us state the so-called intersection problem which gained some attention some time ago. The problem is to establish an identity

$$
(A, B)_{\theta, p} \cap(A, C)_{\theta, p}=(A, B \cap C)_{\theta, p},
$$

where $A, B, C$ are embedded in a common superspace. Generalizing results of Grisvard from [5], Peetre showed in [14] that such an identity holds if one supposes $(A, B)$ to be a "quasilinearizable pair" (see also [15] for the definition of this notion) and an additional assumption. Now, identity (1.5) of Theorem 1.1 shows that also $A=B+C$ is an assumption which renders the intersection identity to be true. This has already been observed in [1]. We can generalize this result towards the condition

$$
B \cap C \subset A \subset(A \cap B)+(A \cap C)
$$

which also implies (3.1).

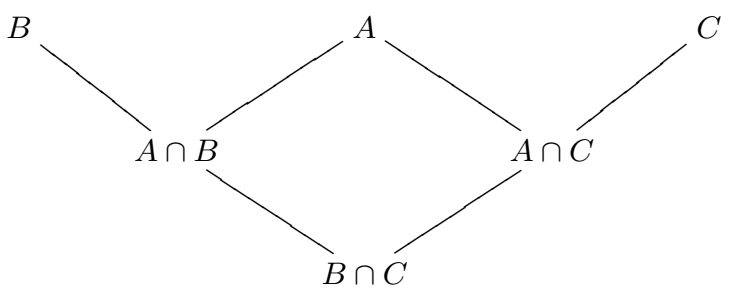

Indeed, it follows first that $(A+B) \cap(A+C)=A$ and this implies $(A, B)_{\theta, p} \cap$ $(A, C)_{\theta, p} \subset A$. Hence,

$$
\begin{aligned}
(A, B)_{\theta, p} & \cap(A, C)_{\theta, p}=\left[(A, B)_{\theta, p} \cap A\right] \cap\left[(A, C)_{\theta, p} \cap A\right] \\
& =(A, A \cap B)_{\theta, p} \cap(A, A \cap C)_{\theta, p}=(A, A \cap B \cap A \cap C)_{\theta, p} \\
& =(A, B \cap C)_{\theta, p},
\end{aligned}
$$

where we used identities (1.1) and (1.5).

3.4. Other K-methods. We stated and proved our results in the case of (ordinary) real interpolation. However with appropriate changes in notation we expect the statements to also hold when one uses generalized K-methods as in [12].

\section{REFERENCES}

[1] Pascal Auscher, Alan McIntosh, and Andrea Nahmod. Holomorphic functional calculi of operators, quadratic estimates and interpolation. Indiana Univ. Math. J., 46(2):375-403, 1997. MR1481596 (98k:47033)

[2] Jöran Bergh and Jörgen Löfström. Interpolation Spaces. An Introduction. Grundlehren der mathematischen Wissenschaften, vol. 223 of Springer-Verlag, Berlin-Heidelberg-New York, 1976. MR0482275 (58:2349)

[3] Yu. A. Brudnyı̆ and N. Ya. Krugljak. Interpolation functors and interpolation spaces. Vol. I, volume 47 of North-Holland Mathematical Library. North-Holland Publishing Co., Amsterdam, 1991. Translated from the Russian by Natalie Wadhwa, with a preface by Jaak Peetre. MR.1107298 (93b:46141)

[4] Giovanni Dore. $H^{\infty}$ functional calculus in real interpolation spaces. II. Studia Math., 145(1):75-83, 2001. MR1828994 (2002c:47034) 
[5] Pierre Grisvard. Interpolation non commutative. Atti Accad. Naz. Lincei Rend. Cl. Sci. Fis. Mat. Natur. (8), 52:11-15, 1972. MR0358337(50:10803)

[6] Bernhard Haak, Markus Haase, and Peer Kunstmann. Perturbation, interpolation and maximal regularity. Adv. Differential Equations, 11(2):201-240, 2006.

[7] Markus Haase. The Functional Calculus for Sectorial Operators. Book manuscript. To appear in: Operator Theory: Advances and Applications, Birkhäuser, Basel. Preliminary version available at http://www . mathematik.uni-ulm.de/m5/haase, 2005.

[8] S. G. Kreĭn, Yu. İ. Petunīn, and E. M. Semënov. Interpolation of linear operators, volume 54 of Translations of Mathematical Monographs. American Mathematical Society, Providence, R.I., 1982. Translated from the Russian by J. Szücs. MR0649411 (84j:46103)

[9] Alessandra Lunardi. Interpolation Theory. Appunti, Scuola Normale Superiore, Pisa., 1999.

[10] Lech Maligranda. The $K$-functional for symmetric spaces. In Interpolation spaces and allied topics in analysis (Lund, 1983), volume 1070 of Lecture Notes in Math., pages 169-182. Springer, Berlin, 1984. MR0760482 (86d:46026)

[11] Lech Maligranda. On commutativity of interpolation with intersection. In Proceedings of the 13th winter school on abstract analysis (Srní, 1985), no. 10, pages 113-118 (1986), 1985. MR0894276 (88j:46067)

[12] Lech Maligranda. Interpolation between sum and intersection of Banach spaces. J. Approx. Theory, 47(1):42-53, 1986. MR0843454 (87k:46151)

[13] Celso Martínez Carracedo and Miguel Sanz Alix. The theory of fractional powers of operators. North-Holland Publishing Co., Amsterdam, 2001. MR.1850825 (2002k:47038)

[14] Jaak Peetre. Über den Durchschnitt von Interpolationsräumen. Arch. Math. (Basel), 25:511513, 1974. MR0383103 (52:3984)

[15] Hans Triebel. Interpolation Theory, Function Spaces, Differential Operators. 2nd rev. a. enl. ed. Leipzig: Barth. 532 p. , 1995. MR1328645 (96f:46001)

Abteilung Angewandte Analysis, Universität Ulm, Helmholtzstrasse 18, D - 89069 Ulm, Germany

Current address: Scuola Normale Superiore di Pisa, Piazza dei Cavalieri 7, I - 56126 Pisa, Italy

E-mail address: haase@mathematik.uni-ulm.de 